\title{
An Inversely Designed Model for Calculating Pull-In Limit and Position of Electrostatic Fixed-Fixed Beam Actuators
}

\author{
Cevher Ak and Ali Yildiz \\ Department of Electrical-Electronics Engineering, Mersin University, 33343, Mersin, Turkey \\ Correspondence should be addressed to Ali Yildiz; aliyildiz99@gmail.com
}

Received 11 April 2014; Revised 20 July 2014; Accepted 20 July 2014; Published 20 August 2014

Academic Editor: Fatih Yaman

Copyright (C) 2014 C. Ak and A. Yildiz. This is an open access article distributed under the Creative Commons Attribution License, which permits unrestricted use, distribution, and reproduction in any medium, provided the original work is properly cited.

This study presents an inverse approach to obtain a relation between applied voltage and displacement of the midpoint of fixedfixed beam actuator. The approach has two main sections. The first one is the inverse design of a model to replace real action of upper beam under electrostatic force. The formula obtained from the first section does not comprise the residual stress and gives very small errors when there is no residual stress on the upper electrode. So, the second part was carried out to add this important system variable into the formula. Likewise, inverse solution was again applied in the later section. The final formula demonstrates that pull-in limit of clamped-clamped actuator is to be at around $40 \%$ of original spacing that is in agreement with simulation and previous experimental results. Its percentage errors are within $2 \%$ when compared with simulations that are based on finite element method (FEM). The results are comparable to numerical solutions received from diverse distributed models which require more calculation power in electrostatic and structural domains. On top of that, our formula is valid for all displacements from original position up to pull-in limit.

\section{Introduction}

Electrostatic MEMS based actuators have been used widely as a sensor due to their higher sensitivity, smaller dimensions, low-power consumption, and easy fabrication with new design possibilities. They have been used as a microelectromechanical varactor [1], as a capacitive pressure sensor for measuring blood pressure for a cardiovascular catheter [2], as a mass sensor [3], as an RF microswitch [4, 5], as an energy harvester for MEMS devices [6], and as small force detection [7].

An electrostatically controlled MEMS based fixed-fixed actuator is made up of two parallel conductive beams; the ground electrode is fabricated on a substrate and not movable and the upper electrode is suspended above it with an initial gap $(g)$ and fixed from both ends. When a voltage difference is applied between bottom and upper electrodes, the middle section of top electrode moves towards bottom electrode due to electrostatic force. The counteract spring force will stop the motion of upper electrode at some equilibrium point. The spring force is a linear function of the movement whereas the electrostatic force increases with the square of distance. After a certain point, the restoring force due to bending cannot balance the electrostatic force any longer. The upper electrode is instable after this point and collides on the bottom electrode. This limit is called pull-in limit and voltage value is named as pull-in voltage. The real behavior of upper electrode can be seen in Figure 1. It obeys two constraints; fixed ends have zero movement and zero-angle.

Calculating the pull-in voltage value accurately is the most crucial issue in MEMS actuators. Nevertheless, behavior is nonlinear because of electrostatic and mechanical coupling. Hence, getting an analytical formula for pull-in limit is very difficult. For more than two decades, researchers have been developing many models and methods for electrostatic MEMS based actuators to calculate pull-in limit [8-16]. However, most of these works have found different pull-in limits. When it is controlled by software (COMSOL) which employs finite element method, pull-in limit appears to be at approximately $40 \%$ of initial gap which is also consistent 


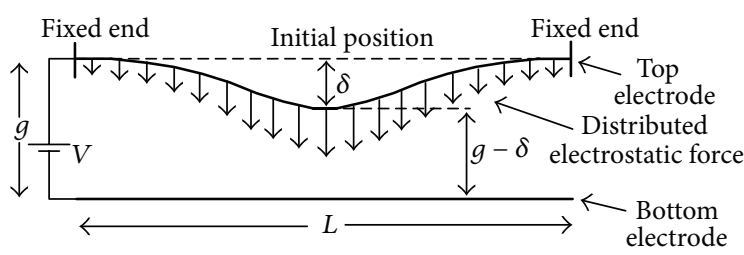

FIGURE 1: Electrostatic actuator (side view).

Table 1: COMSOL Simulation Pull-in Results of a Fixed-Fixed Beam with length $=200 \mu \mathrm{m}$, width $=50 \mu \mathrm{m}$, thickness $=1 \mu \mathrm{m}$, Young Modulus $=160 \mathrm{GPa}$, Poisson Ratio $=0.22$.

\begin{tabular}{lcc}
\hline Initial Gap $(\mu \mathrm{m})$ & Pull-in Gap $(\mu \mathrm{m})$ & Pull-in Gap/Initial Gap \\
\hline 2 & 0.797 & 0.3985 \\
4 & 1.594 & 0.3985 \\
5 & 1.992 & 0.3984 \\
10 & 3.984 & 0.3984 \\
\hline
\end{tabular}

with former experimental measurements [12, 14]. Table 1 points some simulation results out for varying initial gaps. Upper beam was simulated as linear and isotropic poly-Si material in 3D geometry. Its length was chosen as $200 \mu \mathrm{m}$ for simulation purposes only since length is not a function of pull-in gap. Ratio of pull-in gap to initial gap is constant and independent of material properties of the upper electrode. Other properties of the material in simulation can be seen in Table 1.

The main intention of this work is to find a relatively simple formula which demonstrates not only the pull-in limit at the actual position, but also a relationship between applied potential difference and displacement of the middle part of a clamped-clamped electrostatic actuator.

\section{Inversely Designed Model}

We have altered the real behavior of upper electrode with a new model for the sake of simplicity. Upper electrode has subdivided into three regions with four pivots. Two of the pivots are located at both fixed ends. They can only rotate around the pivot points whereas they cannot move horizontally and vertically. The other pivot points are located at $3 L / 7$ distances from both fixed sides symmetrically. These pivots are free to move vertically though. The new model can be seen clearly in Figure 2. Total length of the electrode is $L$. The first and third partitions divided as $3 L / 7$ while the second one as $L / 7$. These values have been inversely reached by trial and error method since we already know the true results from simulations and previous experimental studies.

We had studied bisection model for fixed-free actuators earlier [21, 22]. The model was dividing the upper electrode into two partitions. One is not moving at all and fixed to the substrate from one end and the other section can freely move linearly around a pivot which is selected by trial and error method inversely. It was successful as well when compared with simulation results and experimental studies. Later, model for fixed-free actuators was simplified further

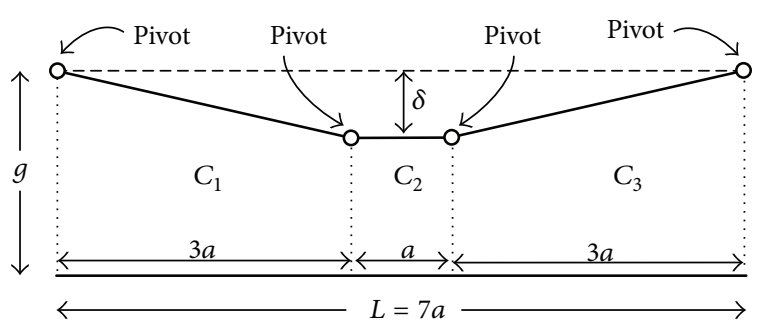

Figure 2: The movements and capacitances of the partitions introduced in new model.

and named as Pivot Model [23]. In the model, fixed side of the upper electrode is pivoted and the other side is totally free to move linearly around the pivot point. The upper beam was assumed as a rigid body in the model for the sake of simplicity. These were reasonable assumptions since the bending of the beam was very small. It establishes a good relation between applied voltage and displacement span from rest position to pull-in limit.

The new model was developed for clamped-clamped actuators and utilizes only one constraint of the fixed ends of top electrode. Fixed ends still have zero displacement while zero-angle constraint is omitted as in Pivot Model. Thus, only one end of the first and third partitions moves linearly around fixed pivot while the second region is totally free to move vertically. The new design model was named as Inverse Pivot Model (IPM) since pivots were used to make the system uncomplicated. In this model, upper electrode is subdivided into three partitions. Locations of the movable pivots were chosen inversely from simulation results and previous experimental measurements. It was also possible to subdivide the beam into more than 3 partitions in order to have a closer representation of the real bending shape of the beam. However, model would get complicated which is not a desired case. In this study, keeping the model as simple as possible is foremost and initiative intention while it gives a good approximation of the real system. After many comparisons with simulation outcomes and previous measurements, length of the upper beam was subdivided into 7 equal segments. One segment was taken as a middle partition, and the other 2 partitions equally shared the rest of the segments due to symmetry. Therefore, side partitions (partitions 1 and 3 ) consist of 3 segments.

The capacitance calculation of the second partition is easy since it establishes a parallel plate capacitance shape during its movement and $C_{2}$ can be calculated as

$$
C_{2}=\frac{1}{7} \frac{\varepsilon_{0} w L}{(g-\delta)}
$$

where $\varepsilon_{0}$ is permittivity of free space. Capacitances of the first and third partitions are equal because of the symmetry. Therefore, calculating one of them will be enough. However, calculation of it is not simple as second partition.

Partial capacitance of $C_{1}$ can be considered as a parallel plate capacitance since the inclination of the upper electrode 


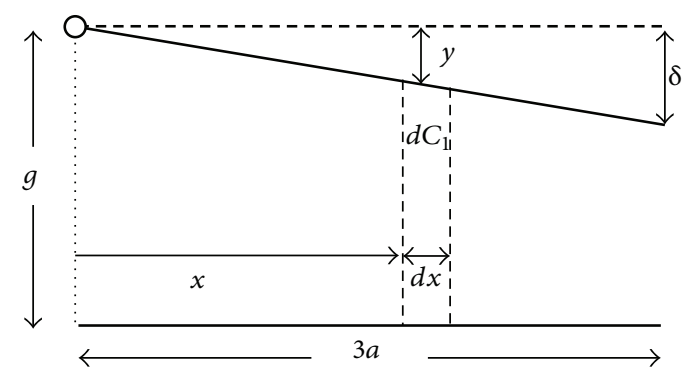

FIgURE 3: Capacitance calculation for the first partition.

is negligible for this infinitesimally small region. It can be seen in Figure 3 and can be written as

$$
d C_{1}=\varepsilon_{0} \frac{d A}{g-y} .
$$

$d A$ is infinitesimal surface area and equal to $w d x$, where $w$ is the width of the upper beam. $y$ can be found from the geometry shown in Figure 3 as

$$
y=\delta \frac{x}{3 a}
$$

where $\delta$ is the farthest displacement of the upper beam when a potential difference is applied. It equals the displacement of middle point as well. Therefore, (2) can be rewritten as

$$
d C_{1}=3 a \varepsilon_{0} \frac{w d x}{3 a g-g x} .
$$

So, capacitances of the first partitions can be calculated as

$$
C_{1}=\int d C_{1}=3 a \varepsilon_{0} w \int_{x=0}^{3 a} \frac{d x}{3 a g-g x} .
$$

When value of $a$ is inserted in (5), $C_{1}$ and $C_{3}$ can be calculated as

$$
C_{1}=C_{3}=\frac{3}{7} \frac{\varepsilon_{0} w L}{\delta} \ln \left(\frac{g}{g-\delta}\right) .
$$

In a real actuator, electrostatic force is nonuniform and distributed along the upper beam. It is extremely difficult to express it in a simple formula. Therefore, it has been exchanged with a single equivalent electrostatic force term. Since the middle region is the closest part of the upper electrode to bottom one, the electrostatic force has the biggest component at this point. Besides, because of symmetry, electrostatic force is placed at the center of the upper electrode as a single equivalent force. Nevertheless, fringing effect of the capacitances was ignored in order to keep the model simple. To compensate the absence of the fringing effect, restoring forces are adjusted accordingly. Instead of using one restoring force term at the center, it has been split into three. Two restoring forces are placed at the one-third of the linearly moving partitions from fixed ends in order to decrease effect of spring force term to make up the missing fringing effect.

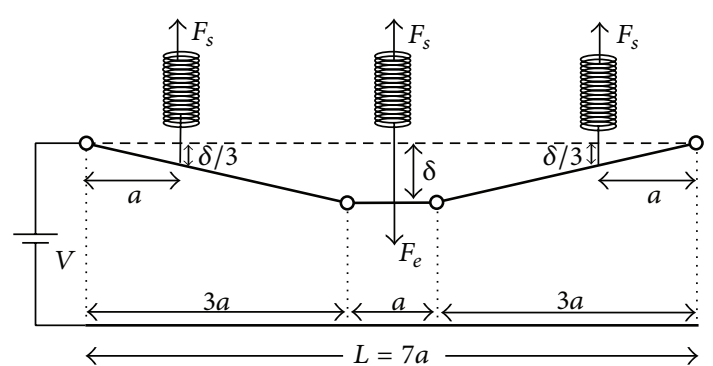

FIGURE 4: Locating the equivalent forces of new model.

The final exact point is again reached after many attempts by inverse approach. Optimization algorithms can also be used to find a more precise location in the future studies. The middle spring force is apparently placed at the center. All equivalent forces and their locations can be seen in Figure 4.

The infinitesimal electrostatic force term can be written as

$$
d F_{e}=\frac{1}{2} \frac{d C}{d \delta} V^{2}
$$

where $C$ is the total capacitance term of the system and $V$ is potential difference applied between electrodes. The total electrostatic force term can be calculated as

$$
\begin{aligned}
F_{e} & =-\frac{\varepsilon_{0} w L}{14} \\
& \times\left(\frac{5 \delta^{2}-6 \delta g+\left(6 \delta^{2}+6 g^{2}-12 \delta g\right) \ln (g /(g-\delta))}{\delta^{2}(g-\delta)^{2}}\right) V^{2}
\end{aligned}
$$

and spring constant $k$ can be obtained for a fixed-fixed beam as [24]

$$
k=32 \widehat{E} w\left(\frac{t}{L}\right)^{3}
$$

where

$$
\widehat{E}=\frac{E}{\left(1-v^{2}\right)}
$$

where $E, v$, and $t$ are Young's modulus, Poisson ratio of material, and the thickness of the upper electrode, respectively. Instead of plain $E, \widehat{E}$ has been used to keep the formula valid for wide beams $w \geq 5 t$ [17].

Since the 3 restoring springs are parallel to each other, spring constant of each partition is equal to $k / 3$. The spring displacement of middle partition is a distance $\delta$, while displacements of the other two partitions are $\delta / 3$. The total restoring force term can be written for 3 partitions as

$$
F_{y}=\delta\left(\frac{k}{3}\right)+2\left(\frac{\delta}{3}\right)\left(\frac{k}{3}\right)=\frac{160}{9} \frac{\delta E w}{\left(1-v^{2}\right)}\left(\frac{t}{L}\right)^{3} \text {. }
$$


TABle 2: Comparison of Voltage Values for Arbitrary Displacements. $E=169 \mathrm{GPa}, L=350 \mu \mathrm{m}, t=3 \mu \mathrm{m}, \nu=0.06$ and $g=1 \mu \mathrm{m}$.

\begin{tabular}{lccc}
\hline $\begin{array}{l}\text { Displacement } \\
(\mu \mathrm{m})\end{array}$ & $\begin{array}{c}\text { Voltage (Volt) } \\
\text { IPM }(9)\end{array}$ & $\begin{array}{c}\text { Voltage (Volt) } \\
\text { COMSOL }\end{array}$ & $\Delta \%$ \\
\hline 0.0116 & 4.9518 & 5 & 0.97 \\
0.0493 & 9.9076 & 10 & 0.93 \\
0.0739 & 11.8935 & 12 & 0.90 \\
0.1062 & 13.8822 & 14 & 0.85 \\
0.1496 & 15.8724 & 16 & 0.80 \\
0.1775 & 16.8617 & 17 & 0.82 \\
0.2125 & 17.8567 & 18 & 0.80 \\
0.2594 & 18.8394 & 19 & 0.85 \\
0.3445 & 19.8053 & 20 & 0.98 \\
0.3891 & 19.9634 & 20.19 & 1.14 \\
\hline
\end{tabular}

The first term in (11) represents the restoring force of the middle partition spring, and the second term represents the total restoring forces of the side partitions springs.

Since the electrostatic and restoring forces have to be equal to each other at equilibrium position, (8) and (11) can be equated to each other as

$$
\begin{aligned}
& -\frac{\varepsilon_{0} w L}{14}\left(\frac{5 \delta^{2}-6 \delta g+\left(6 \delta^{2}+6 g^{2}-12 \delta g\right) \ln (g /(g-\delta))}{\delta^{2}(g-\delta)^{2}}\right) \\
& \times V^{2}=\frac{160}{9} \frac{\delta E w}{\left(1-v^{2}\right)}\left(\frac{t}{L}\right)^{3} .
\end{aligned}
$$

Consequently, the relation between displacement and applied voltage can be obtained from (12) as

$$
\begin{aligned}
V=\left(\frac{2240}{9}(-\right. & E t^{3} \delta^{3}(\delta-g)^{2} \\
& \times\left(\varepsilon_{0} L^{4}\left(1-v^{2}\right)\right. \\
& \times\left(\ln \left(\frac{g}{g-\delta}\right)\left(6 \delta^{2}+6 g^{2}-12 \delta g\right)\right. \\
& \left.\left.\left.\left.+5 \delta^{2}-6 \delta g\right)\right)^{-1}\right)\right)^{1 / 2} .
\end{aligned}
$$

Equation (13) is valid not only for pull-in limit, but also for all values within the pull-in limit. A comparison of simulation results received from COMSOL package and our IPM results for different displacements up to pull-in limit boundary. All results seem within $1 \%$ error level.

Many fixed-fixed actuator sensors employ only on/off position like a switch. Therefore, determination of pull-in voltage is very crucial for an actuator before real device fabrication in order to produce a proper sensor which is working in a range of interest. In order to retrieve the pullin limit, derivative of $V$ in (13) with respect to $\delta$ has to
TABLE 3: Comparison of IPM and IPMF with previous simulation results. $L=250 \mu \mathrm{m}, E=169 \mathrm{GPa}, \nu=0.06, w=50 \mu \mathrm{m}, t=3 \mu \mathrm{m}$ and $g=1 \mu \mathrm{m}$ in each case.

\begin{tabular}{lccc}
\hline $\begin{array}{l}\text { Residual Stress, } \sigma, \\
(\mathrm{MPa})\end{array}$ & 0 & 100 & -25 \\
\hline CoSolve [17] & 40.10 & 57.60 & 33.60 \\
V (2D) [17] $(\Delta \%)$ & $39.50(1.50 \%)$ & $56.90(1.22 \%)$ & $33.70(0.30 \%)$ \\
OCI Model [12] $(\Delta \%) 39.60(1.25 \%)$ & $57.40(0.35 \%)$ & $33.71(0.33 \%)$ \\
GDQM [15] $(\Delta \%)$ & $39.13(2.41 \%)$ & $57.62(0.03 \%)$ & $33.63(0.09 \%)$ \\
IPM $(\Delta \%)$ & $39.13(2.41 \%)$ & $39.13(32.07 \%)$ & $39.13(16.461 \%)$ \\
IPMF $(\Delta \%)$ & $39.42(1.70 \%)$ & $57.03(0.99 \%)$ & $33.61(0.03 \%)$ \\
\hline
\end{tabular}

be taken and equate the result to zero and this position is named as pull-in limit [9]. The upper beam will be unstable and collapse towards bottom beam after this critical point. Since the derivative equation equals zero, we can use only the numerator of the derivative since denominator is not affecting the result. So, denominator is dropped from the derivative equation. Subsequently, some common variables are also canceled in order to keep the calculation simple:

$$
18 \ln \left(\frac{g}{g-\delta}\right)(g-\delta)^{3}-\delta(2 g-3 \delta)(9 g-7 \delta)=0 .
$$

Unfortunately, having an analytical solution to this equation is very cumbersome. Thence, a computational result has been obtained by iteration as

$$
\delta_{\text {Pull-in Limit }} \cong 0.4 g \text {. }
$$

This value also can be seen in Table 2 . When $\delta / g$ gets closer to 0.4 , voltage value starts to saturate. When this important value was inserted into (13) back, crucial value of potential difference can be found as

$$
V_{\text {Pull-in Limit }}=\sqrt{11.5486 \frac{E g^{3} t^{3}}{\left(1-v^{2}\right) \varepsilon_{0} L^{4}}} \text {. }
$$

This value is the potential difference value at the pull-in limit boundary. Applying a voltage difference higher than this value causes the upper electrode to be unstable and collapse onto the bottom electrode.

\section{Inverse Pivot Modified Formula}

When it is checked with simulation results obtained by a different software package which utilize finite element method from a previous study, it can be seen that formula gives small errors if there is no residual stress. However, error goes as high as $45 \%$ whenever residual stress gets involved since our Inverse Pivot Model does not take residual stress into account. Effect of residual stress can be seen in Table 3 . Thence, we decided to improve the formula by considering residual stress as a system parameter. In this second section, formula is merely modified inversely since we have a starting point (equation (16)) and the true results from previous 
TABLE 4: Comparison of IPM and IPMF with previous simulation results. $L=350 \mu \mathrm{m}, E=169 \mathrm{GPa}, \nu=0.06, w=50 \mu \mathrm{m}, t=3 \mu \mathrm{m}$ and $g=1 \mu \mathrm{m}$ in each case.

\begin{tabular}{lccc}
\hline $\begin{array}{l}\text { Residual Stress, } \sigma, \\
(\mathrm{MPa})\end{array}$ & 0 & 100 & -25 \\
\hline CoSolve [1] & 20.30 & 35.80 & 13.70 \\
V (2D) [17] $(\Delta \%)$ & $20.20(0.50 \%)$ & $35.40(1.11 \%)$ & $13.80(0.73 \%)$ \\
OCI Model [12] $(\Delta \%) 20.20(0.50 \%)$ & $35.91(0.31 \%)$ & $13.71(0.07 \%)$ \\
GDQM [15] $(\Delta \%)$ & $20.36(0.30 \%)$ & $35.99(0.53 \%)$ & $13.60(0.73 \%)$ \\
IPM $(\Delta \%)$ & $19.96(1.67 \%)$ & $19.96(44.25 \%)$ & $19.96(45.69 \%)$ \\
IPMF $(\Delta \%)$ & $20.11(0.94 \%)$ & $35.25(1.54 \%)$ & $13.97(1.97 \%)$ \\
\hline
\end{tabular}

researches $[12,15,17]$ to compare outcomes of the new formula. Adding residual stress into (16) is easier than starting from scratch to get a new model. From Tables 3 and 4, it can be apparently seen that positive residual stress increases whereas negative one decreases pull-in voltage. Nevertheless, effect of residual stress should increase with the thickness and decrease with the length of upper electrode. We had to move some physical and system parameters from numerator to denominator in order to keep formula dimensionally correct. After many trials, Inverse Pivot Modified Formula (IPMF) has been reached as

$$
V=\left(\frac{\left(\sigma t(t / L)^{1.1}+(65 / 9)\left(E g t^{3} /\left(1-v^{2}\right) L^{3}\right)\right)}{(61 / 99)\left(\varepsilon_{0} L / g^{2}\right)}\right)^{1 / 2},
$$

where $\sigma$ is the residual stress. Equation (17) has not been attained as an analytical calculation. However, when the residual stress is assumed as zero in this equation, it closely approaches (16). It also establishes very good results for actuators with residual stress at pull-in limit boundary. Errors are now in acceptable ranges which all are smaller than $2 \%$, even for the worst case (see Tables 3 and 4).

In Tables 3 and 4, Poisson ratios are 0.06 in all cases. So, our model was also checked with a different Poisson ratio which is 0.32 . From Table 5, it can be seen that IPM delivers a comparable result with other studies. In particular, IPMF demonstrates very similar error levels when compared with previous methods which depends on numerical distributed models. These kinds of model require more computing power and need more time to get the result. However, our formula is just one step calculation if it is written in an Excel sheet.

The IPMF is also checked for some other parameters in order to explore the applicable range of the formula. Table 6 shows simulation results obtained in COMSOL for a poly-Si beam whose material properties are selected from COMSOL's library. Mesh numbers are selected automatically for each case in the software.

Table 6 demonstrates comparison between IPMF and simulation results for wide range of material parameters. IPMF gives very satisfactory results with a maximum error level of $8 \%$ for residual stress case. In Table 7 , width is changed for $200 \mu \mathrm{m}$ and $500 \mu \mathrm{m}$ beam lengths while other parameters are kept constant to show the effect of width. IPMF again shows very satisfactory results for the range of
TABLE 5: Comparison of IPM and IPMF with previous simulation results [12]. $L=250 \mu \mathrm{m}, E=169 \mathrm{GPa}, v=0.32, w=50 \mu \mathrm{m}, t=$ $3 \mu \mathrm{m}$ and $g=1 \mu \mathrm{m}$ in each case.

\begin{tabular}{lc}
\hline Residual Stress, $\sigma,(\mathrm{MPa})$ & 0 \\
\hline CoSolve FEA [17] & 41.20 \\
V $(2 \mathrm{D})[17](\Delta \%)$ & $41.50(0.73 \%)$ \\
{$[18](\Delta \%)$} & $42.54(3.25 \%)$ \\
{$[19](\Delta \%)$} & $41.20(0.00 \%)$ \\
{$[20](\Delta \%)$} & $41.42(0.53 \%)$ \\
OCI Model [12] & $41.72(1.26 \%)$ \\
IPM $(\Delta \%)$ & $41.53(0.80 \%)$ \\
IPMF $(\Delta \%)$ & $41.23(0.07 \%)$ \\
\hline
\end{tabular}

TABLE 6: Pull-in Voltage Comparison of IPMF to simulation results for Poly-Si material from COMSOL library. $E=160 \mathrm{GPa}, v=0.22$ and $w=50 \mu \mathrm{m}$ for all cases.

\begin{tabular}{|c|c|c|c|}
\hline Residual Stress, $\sigma,(\mathrm{MPa})$ & COMSOL $(\mathrm{V})$ & $\operatorname{IPMF}(\mathrm{V})$ & $(\Delta \%)$ \\
\hline \multicolumn{4}{|c|}{$L=100 \mu \mathrm{m}, t=2 \mu \mathrm{m}, g=1 \mu \mathrm{m}$, Number of Meshes $=7533$} \\
\hline 0 & 134.70 & 132.53 & 1.61 \\
\hline-25 & 130.30 & 127.74 & 1.96 \\
\hline 100 & 150.00 & 150.18 & 0.12 \\
\hline \multicolumn{4}{|c|}{$L=200 \mu \mathrm{m}, t=2 \mu \mathrm{m}, g=1 \mu \mathrm{m}$, Number of Meshes $=4467$} \\
\hline 0 & 33.60 & 33.13 & 1.40 \\
\hline-25 & 29.00 & 28.40 & 2.07 \\
\hline 100 & 47.50 & 47.55 & 0.11 \\
\hline \multicolumn{4}{|c|}{$L=300 \mu \mathrm{m}, t=3 \mu \mathrm{m}, g=2 \mu \mathrm{m}$, Number of Meshes $=3531$} \\
\hline 0 & 78.30 & 76.52 & 2.27 \\
\hline-25 & 67.90 & 68.49 & 0.87 \\
\hline 100 & 109.00 & 102.51 & 5.95 \\
\hline \multicolumn{4}{|c|}{$L=400 \mu \mathrm{m}, t=3 \mu \mathrm{m}, g=2 \mu \mathrm{m}$, Number of Meshes $=2817$} \\
\hline 0 & 44.01 & 43.04 & 2.20 \\
\hline-25 & 34.00 & 34.88 & 2.59 \\
\hline 100 & 72.00 & 66.30 & 7.92 \\
\hline \multicolumn{4}{|c|}{$L=500 \mu \mathrm{m}, t=4 \mu \mathrm{m}, g=2 \mu \mathrm{m}$, Number of Meshes $=3259$} \\
\hline 0 & 42.83 & 42.41 & 0.98 \\
\hline-25 & 33.30 & 32.71 & 1.77 \\
\hline 100 & 68.10 & 68.64 & 0.79 \\
\hline \multicolumn{4}{|c|}{$L=750 \mu \mathrm{m}, t=5 \mu \mathrm{m}, g=3 \mu \mathrm{m}$, Number of Meshes $=1782$} \\
\hline 0 & 49.10 & 48.39 & 1.45 \\
\hline-25 & 32.80 & 34.99 & 6.68 \\
\hline 100 & 87.00 & 82.53 & 5.14 \\
\hline
\end{tabular}

width with a maximum error level of 3.5\%. In Table 8, initial gap is changed for again $200 \mu \mathrm{m}$ and $500 \mu \mathrm{m}$ beam lengths while other parameters are kept constant to show the effect of the gap between electrodes. It can be seen that error level gets increase as the initial gap heightens for both $200 \mu \mathrm{m}$ and $500 \mu \mathrm{m}$ beam lengths. This error stems from ignorance of fringing effect between upper and bottom electrodes. As the initial gap rises, the capacitance value of the system also proliferates due to additional increase of the fringing effect. 
TABLE 7: Comparison of IPMF to simulation results for the variety of widths. $E=160 \mathrm{GPa}, \nu=0.22$ and $w=50 \mu \mathrm{m}$ for all cases.

\begin{tabular}{lccc}
\hline Width, $w,(\mu \mathrm{m})$ & COMSOL $(\mathrm{V})$ & $\operatorname{IPMF}(\mathrm{V})$ & $(\Delta \%)$ \\
\hline \multicolumn{4}{c}{$L=200 \mu \mathrm{m}, t=2 \mu \mathrm{m}, g=1 \mu \mathrm{m}$} \\
50 & 33.60 & 33.13 & 1.40 \\
20 & 33.30 & 33.13 & 0.51 \\
10 & 33.10 & 33.13 & 0.09 \\
5 & 32.90 & 33.13 & 0.70 \\
2 & 32.60 & 33.13 & 1.63 \\
1 & 32.20 & 33.13 & 2.89 \\
\hline & $4=500 \mu \mathrm{m}, t=4 \mu \mathrm{m}, g=2 \mu \mathrm{m}$ \\
50 & 42.83 & 42.41 & \\
20 & 42.30 & 42.41 & 0.98 \\
10 & 42.10 & 42.41 & 0.26 \\
5 & 41.80 & 42.41 & 0.74 \\
2 & 41.40 & 42.41 & 1.46 \\
1 & 41.00 & 42.41 & 2.44 \\
\hline
\end{tabular}

\section{Conclusions}

In the present study, we have proposed a new inversely designed model (IPM) and modified formula (IPMF) which both deliver pull-in limit of a fixed-fixed beam actuator at around $40 \%$ of the initial gap. They are consistent with COMSOL simulation results (Tables 1 and 2) and previous experimental and distributed models (Tables 3, 4, and 5). Besides, it establishes a good relation between applied voltage and displacements commencing from rest position up to pullin limit. Particularly, our inversely designed modified formula yields percentage errors less than $2 \%$, even for the worst case. IPMF is a plain formula which does not demand much computing power. Moreover, IPM is successful with gratifying outcomes for applied voltages for given displacements. When compared with previous experimental measurements, IPM and IPMF can be found considerably successful too. However, if the upper electrode has residual stress, our first model IPM cannot deliver good results because model does not take residual stress into account. On the other hand, IPMF still demonstrates very small percentage errors which are comparable to previous distributed models. IPMF also attests successful outcomes for a wide range of top beam geometric parameters (Tables 6 and 7). The formula is valid for both long beams where $L \geq 5 w$ and narrow beams $(w>$ $5 t)[12]$.

The most eminent benefit of IPMF is that it establishes a good approximation of the system with an unsophisticated plain formula. One can simply calculate required voltage for pull-in limit rather than utilize numerical distributed methods which requires more computing power and is time consuming.

Although IPM and IPMF are outstandingly precise especially around pull-in limit, they have some limitations since they neglect some physical parameters of the system for sake of simplification of the final formula. Firstly, both of them do not take the fringing effect into account. It causes the actual capacitance to be higher than what we have calculated
TABLE 8: Comparison of IPMF to simulation results for the variety of initial gaps. $E=160 \mathrm{GPa}, \nu=0.22$ and $w=50 \mu \mathrm{m}$ for all cases.

\begin{tabular}{lccc}
\hline Gap, $g,(\mu \mathrm{m})$ & COMSOL $(\mathrm{V})$ & $\operatorname{IPMF}(\mathrm{V})$ & $(\Delta \%)$ \\
\hline \multicolumn{4}{c}{$L=200 \mu \mathrm{m}, w=50 \mu \mathrm{m}, t=2 \mu \mathrm{m}$} \\
1 & 33.60 & 33.13 & 1.40 \\
2 & 99.50 & 93.71 & 5.82 \\
3 & 196.00 & 172.17 & 12.16 \\
5 & 520.00 & 370.45 & 28.76 \\
\hline & $L=500 \mu \mathrm{m}, w=50 \mu \mathrm{m}, t=4 \mu \mathrm{m}$ \\
2 & 42.83 & 42.41 & \\
3 & 79.80 & 77.91 & 0.98 \\
4 & 126.00 & 119.95 & 2.37 \\
6 & 249.00 & 220.38 & 4.81 \\
\hline
\end{tabular}

in our Inverse Pivot Model. Therefore, when the initial gap gets higher, IPMF also deviates from acceptable error levels (Table 8).

Secondly, the upper beam was also assumed as a rigid body in the model. Thence, length of the beam was taken as constant even under significant electrostatic force. Therefore, both axial and transverse stresses would not be formed.

Lastly, the models assume the actuators are in vacuum since they ignore any atmospheric pressure on the upper electrode.

IPMF can be improved more by applying artificial optimization techniques and fringing effect of the capacitance geometry can be taken into account to make the formula deliver smaller error level.

\section{Conflict of Interests}

The authors declare that there is no conflict of interests regarding the publication of this paper.

\section{Acknowledgment}

The authors would like to thank the anonymous reviewers who have helped to improve the quality of their paper.

\section{References}

[1] A. L. Roy, A. Bhattacharya, R. R. Chaudhuri, and T. K. Bhattacharyya, "Analysis of the pull-in phenomenon in microelectromechanical varactors," in Proceedings of the 25th International Conference on VLSI Design, pp. 185-190, Hyderabad, India, January 2012.

[2] H.-L. Chau and K. D. Wise, "Ultraminiature solid-state pressure sensor for a cardiovascular catheter," IEEE Transactions on Electron Devices, vol. 35, no. 12, pp. 2355-2362, 1988.

[3] W. Zhang, R. Baskaran, and K. L. Turner, "Effect of cubic nonlinearity on auto-parametrically amplified resonant MEMS mass sensor," Sensors and Actuators A, vol. 102, no. 1-2, pp. 139150, 2002.

[4] S. P. Pacheco, L. P. B. Katehi, and C. T.-C. Nguyen, "Design of low actuation voltage RF MEMS switch," in Proceedings of the IEEE MTT-S International Microwave Symposium Digest, pp. 165-168, Boston, Mass, USA, June 2000. 
[5] H.-H. Yang, O. L. Jeong, and J.-B. Yoon, "Maneuvering pullin voltage of an electrostatic micro-switch by introducing a pre-charged electrode," in Proceedings of the IEEE International Electron Devices Meeting (IEDM '07), pp. 439-442, Washington, DC, USA, December 2007.

[6] D. Shen, J. Park, J. Ajitsaria, S. Choe, H. C. Wikle, and D. Kim, "The design, fabrication and evaluation of a MEMS PZT cantilever with an integrated Si proof mass for vibration energy harvesting," Journal of Micromechanics and Microengineering, vol. 18, no. 5, Article ID 055017, 2008.

[7] P. Mohanty, D. A. Harrington, and M. L. Roukes, "Measurement of small forces in micron-sized resonators," Physica B: Condensed Matter, vol. 284-288, part 2, pp. 2143-2144, 2000.

[8] M. Mojahedi, M. Moghimi Zand, and M. T. Ahmadian, "Static pull-in analysis of electrostatically actuated microbeams using homotopy perturbation method," Applied Mathematical Modelling, vol. 34, no. 4, pp. 1032-1041, 2010.

[9] G. N. Nielson and G. Barbastathis, "Dynamic pull-in of parallelplate and torsional electrostatic MEMS actuators," Journal of Microelectromechanical Systems, vol. 15, no. 4, pp. 811-821, 2006.

[10] Y. Hu and G. Lee, "A closed form solution for the pull-in voltage of the micro bridge," Tamkang Journal of Science and Engineering, vol. 10, no. 2, pp. 147-150, 2007.

[11] L. Mol, E. Cretu, L. A. Rocha, and R. F. Wolffenbuttel, "Fullgap positioning of parallel-plate electrostatic MEMS using onoff control," in Proceedings of the IEEE International Symposium on Industrial Electronics (ISIE '07), pp. 1464-1468, Vigo, Spain, June 2007.

[12] S. Chowdhury, M. Ahmadi, and W. C. Miller, "Pull-in voltage study of electrostatically actuated fixed-fixed beams using a VLSI on-chip interconnect capacitance model," Journal of Microelectromechanical Systems, vol. 15, no. 3, pp. 639-651, 2006.

[13] K. O. Owusu and F. L. Lewis, "Solving the "pull-in" instability problem of electrostatic microactuators using nonlinear control techniques," in Proceedings of the 2nd IEEE International Conference on Nano/Micro Engineered and Molecular Systems (IEEE NEMS '07), pp. 1190-1195, Bangkok, Thailand, January 2007.

[14] Y. C. Hu, C. M. Chang, and S. C. Huang, "Some design considerations on the electrostatically actuated microstructures," Sensors and Actuators A: Physical, vol. 112, no. 1, pp. 155-161, 2004.

[15] H. Sadeghian, G. Rezazadeh, and P. M. Osterberg, "Application of the generalized differential quadrature method to the study of pull-in phenomena of MEMS switches," Journal of Microelectromechanical Systems, vol. 16, no. 6, pp. 1334-1340, 2007.

[16] H. C. Nathanson, W. E. Newell, R. A. Wickstrom, and J. R. Davis Jr., "The resonant gate transistor," IEEE Transaction of Electron Devices, vol. 14, no. 3, pp. 117-133, 1967.

[17] P. M. Osterberg and S. D. Senturia, "M-test: a test chip for MEMS material property measurement using electrostatically actuated test structures," Journal of Microelectromechanical Systems, vol. 6, no. 2, pp. 107-118, 1997.

[18] S. Pamidighantam, R. Puers, K. Baert, and H. A. C. Tilmans, "Pull-in voltage analysis of electrostatically actuated beam structures with fixed-fixed and fixed-free end conditions," Journal of Micromechanics and Microengineering, vol. 12, no. 4, pp. 458-464, 2002.

[19] C. O’Mahony, M. Hill, R. Duane, and A. Mathewson, “Analysis of electromechanical boundary effects on the pull-in of micromachined fixed-fixed beams," Journal of Micromechanics and Microengineering, vol. 13, no. 4, pp. S75-S80, 2003.

[20] H. A. C. Tilmans and R. Legtenberg, "Electrostatically driven vacuum-encapsulated polysilicon resonators. Part II. Theory and performance," Sensors and Actuators A, vol. 45, no. 1, pp. 67-84, 1994.

[21] A. Yildiz, C. Ak, and H. Canbolat, "New approach to pull-in limit and position control," in Electrostatics, chapter 6, pp. 139150, In-Teh, Rijeka, Croatia, 2012.

[22] C. Ak, Dynamic position control of electrostatic actuators [M.S. thesis], Fen Bilimleri Enstitusu, Mersin University, Mersin, Turkey, 2008.

[23] C. Ak and A. Yildiz, "Development of a novel analytical model for calculating pull-in limit and voltage value for a desired position of electrostatic cantilever free tip," Pensee Journal, vol. 76, pp. 360-373, 2014.

[24] S. C. Saha, U. Hanke, G. U. Jensen, and T. Saether, "Modeling of spring constant and pull-down voltage of non uniform RF MEMS cantilever," in Proceedings of the IEEE International Behavioral Modeling and Simulation Workshop, pp. 56-60, September 2006. 


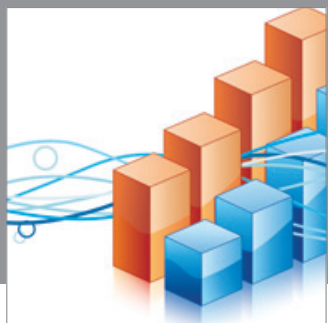

Advances in

Operations Research

mansans

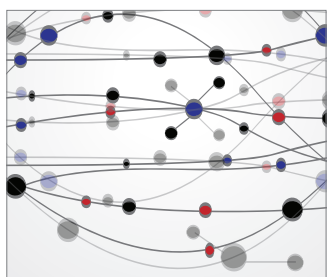

The Scientific World Journal
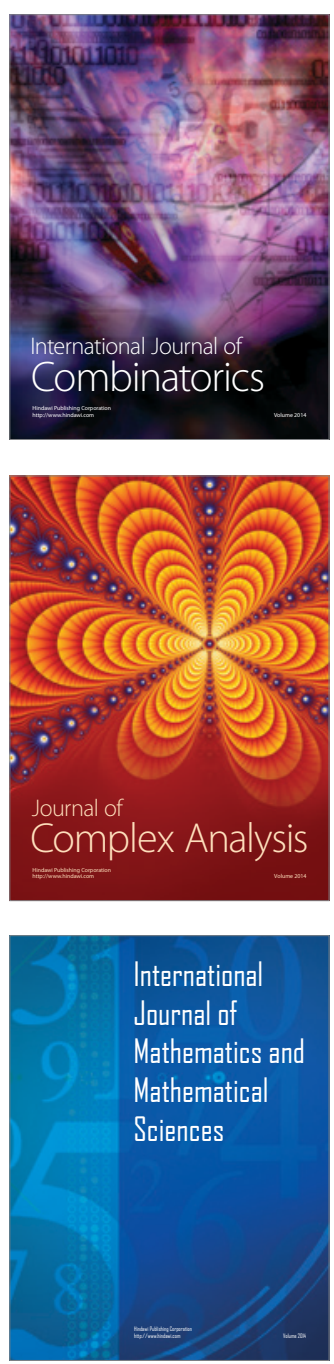
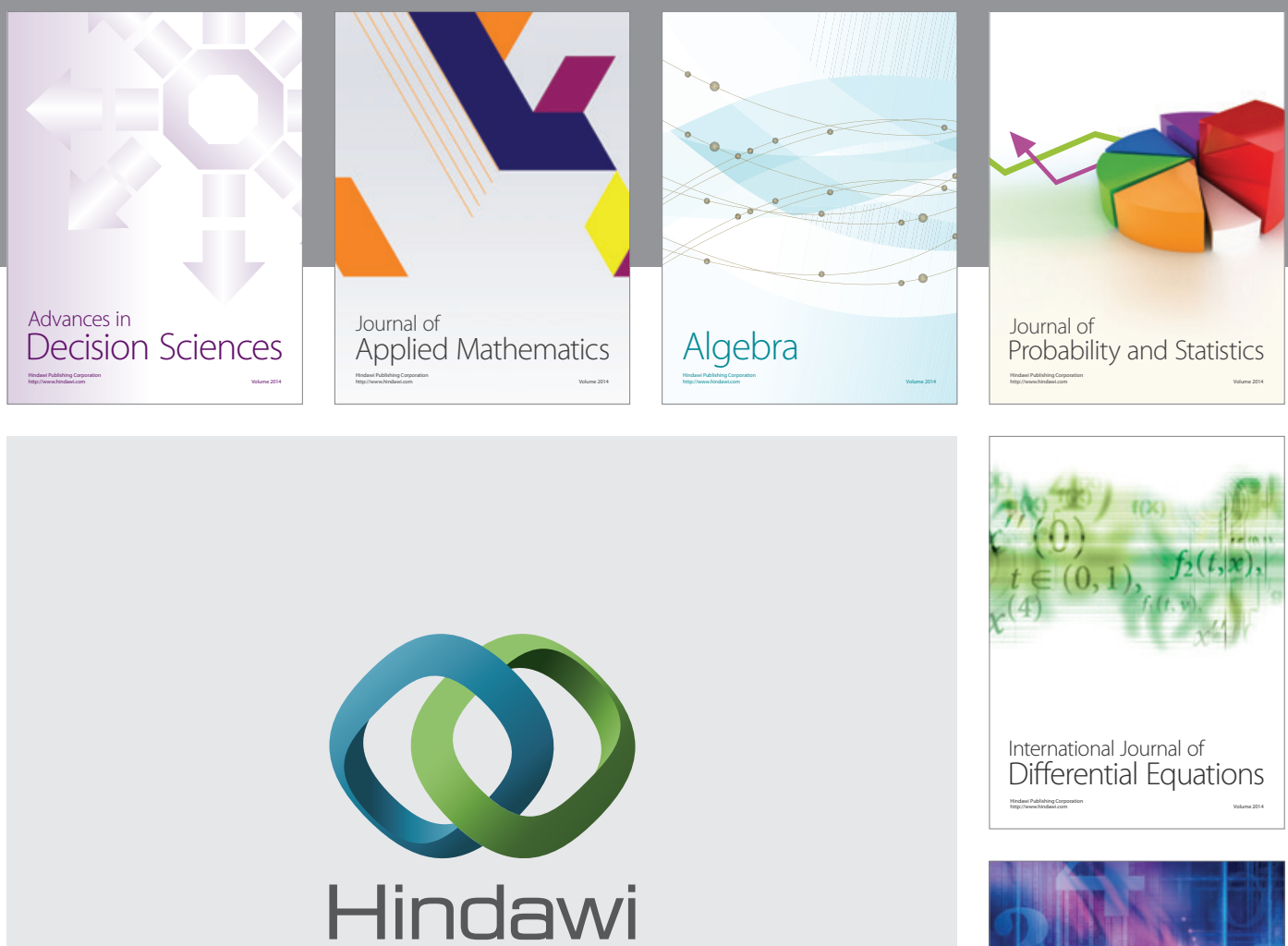

Submit your manuscripts at http://www.hindawi.com
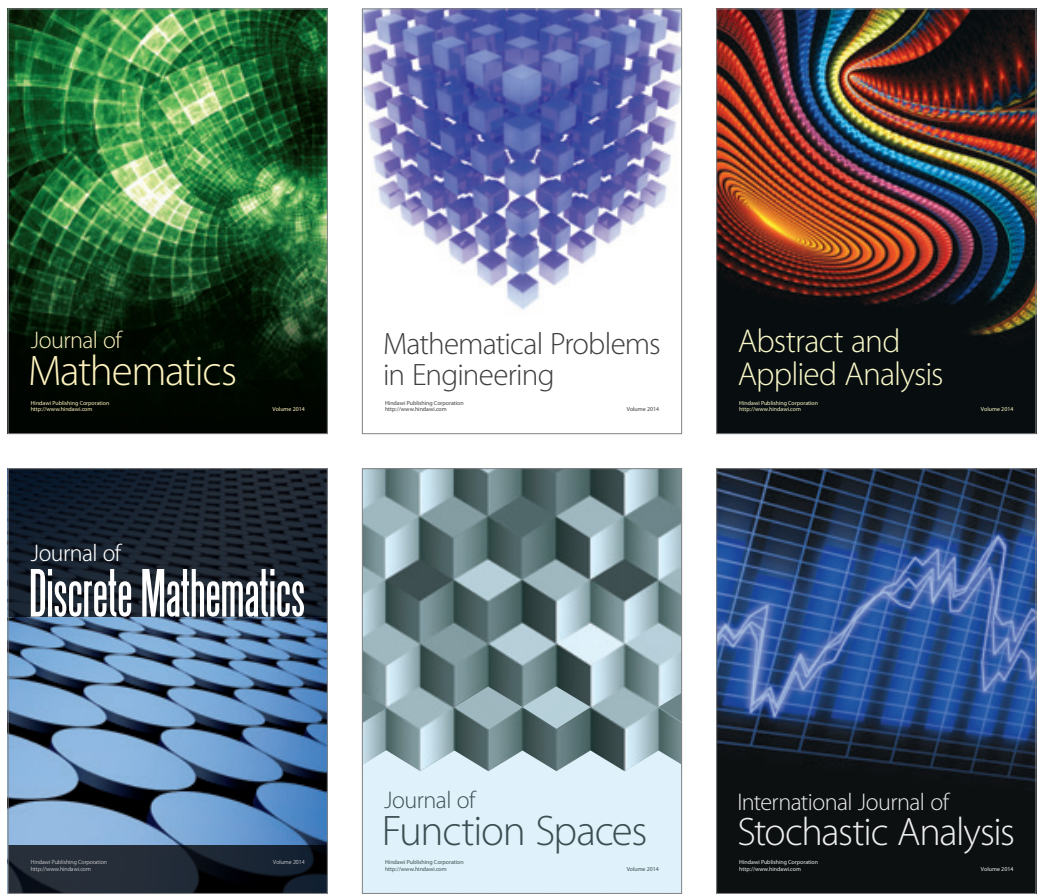

Journal of

Function Spaces

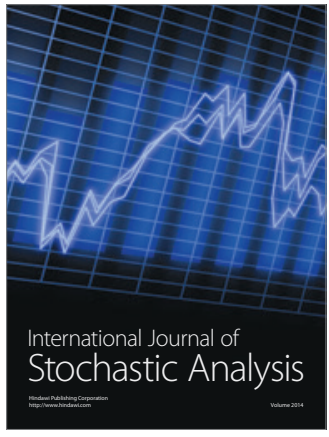

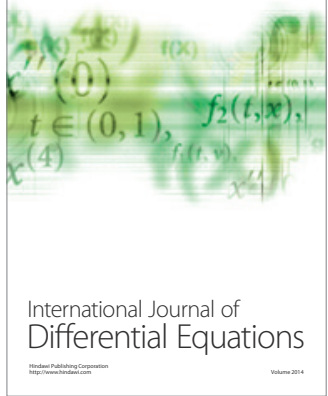
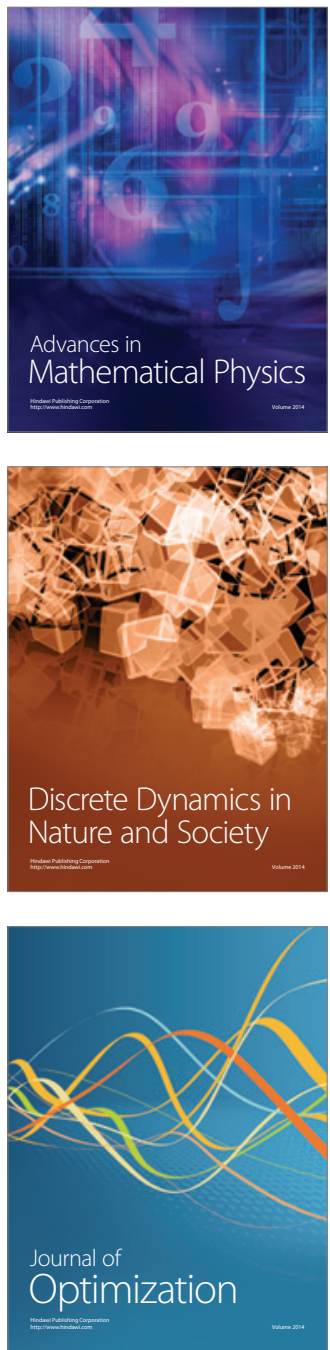